\title{
EL ENFOQUE DE COMPETENCIA Y LOS PROCESOS EDUCATIVOS EN LA INSTITUCIÓN UNIVERSITARIA ANTONIO JOSÉ CAMACHO (UNIAJC): UNA LECTURA CRÍTICA Y UNA HIPÓTESIS DE TRABAJO CURRICULAR INNOVADORA Y ALTERNATIVA A LA CONCEPCIÓN Y PRÁCTICA EN USO
}

\section{PRIMERA PARTE}

Hernán Mera Borrero

Asesor Pedagógico

Institución Universitaria Antonio José Camacho

"Amamos las cadenas, los amos, las seguridades,
Porque nos evitan la angustia de la razón".
Estanislao Zuleta (2015)
"No lo podemos reunir. Está unido".

Torp, L. \& Sage (2007)

Recepción: 08/02/2019. Aceptado: 14/05/2019.

Cómo citar este artículo:

Mera Borrero, H. (2019). El enfoque de competencia y los procesos educativos en la Institución Universitaria Antonio José Camacho (UNIAJC): una lectura crítica y una hipótesis de trabajo curricular innovadora y alternativa a la concepción y práctica en uso. Revista Sapientía, 11 (21), 32 - 45.

\section{RESUMEN}

El artículo socializa reflexiones críticas sobre el tema de la educación por competencias, realizadas desde 1995, una vez se introdujeron en Colombia, a través del SENA (1995-1996), y posteriormente, después del 2000, en el sistema educativo internacional. En la Institución el tema es objeto de examen, primero en el contexto del proyecto Papyrus (2008-2010) y desde 2012, una vez se emprendió la elaboración del Modelo Pedagógico (2013) y, más recientemente, en el 2017, con el proceso de Resignificación Curricular, el que tomó como uno de sus referentes el Enfoque de Competencia asumido por la UNIAJC (2016). Momentos históricos que se constituyen aquí sólo en un eslabón de anclaje que le da continuidad a la reflexión posterior. Desde esta mirada, se revelan las consideraciones conceptuales como preámbulo, justificación y acceso al nuevo abordaje que se propone. En efecto, la atención se centra en la problemática educativa que trae o encubre la palabra, en la innovación que podría introducir o no en los procesos formativos y, a su vez, se invita al estudio y al consecuente debate académico que es necesario en las actuales condiciones educativas que viven el mundo y el país, que tiene que ver con las grandes inquietudes que ha suscitado el término competencia tanto en su inserción en el campo laboral como educativo, en específico en la universidad. Por otra parte, en este contexto problemático de discusión, emerge la necesidad, determinada a la vez por razones educativas teóricas y prácticas, sociales, económicas y políticas, de reinventar la educación, el modelo de universidad colombiano, donde la relación sociedad, universidad y conocimiento es de la mayor importancia. 


\section{PALABRAS CLAVE}

Competencias, enfoque de competencia, resignificación curricular, praxis educativa, contradicción dialéctica, fenómeno/esencia, obstáculo epistemológico, complejidad, pensar complejo, pensar sistémico.

\section{ABSTRACT}

The article socializes critical reflections on the issue of education by competences, made since 1995, once they were introduced in Colombia, through the SENA (1995-1996), and later, after 2000, in the international education system. In the Institution, the subject is under examination, first in the context of the Papyrus project (2008-2010) and since 2012, once the Pedagogical Model was developed (2013) and, more recently, in 2017, with the process of Curricular Resignification, which took as one of its referents the Competency Approach assumed by the UNIAJC (2016). Historical moments that constitute here only a link of mooring that gives continuity to the later reflection. From this perspective, conceptual considerations are revealed as a preamble, justification and access to the new approach proposed. In effect, attention is focused on the educational problem that brings or conceals the word, on the innovation that may or may not introduce the training processes and, at the same time, invites the study and consequent academic debate that is necessary in the present educational conditions that the world and the country are experiencing, which has to do with the great concerns that the term competition has aroused both in its insertion in the labor and educational fields, specifically in the university. On the other hand, in this problematic context of discussion, emerges the need, determined at the same time for theoretical and practical educational, social, economic and political reasons, to reinvent education, the model of Colombian university, where the relationship between society, university and Knowledge is of the greatest importance.

\section{KEYWORDS}

Competencies, competency approach, curriculum resignification, educational practice, dialectical contradiction, phenomenon/essence, epistemological obstacle, complexity, think complex, think systemic.

\section{INTRODUCCIÓN}

Indudablemente uno de los temas más controversiales en el ámbito educativo es el relacionado con los procesos formativos asociados al desarrollo de competencias. Y esta cobra especial relevancia en el contexto de la educación superior, por varias razones como veremos más adelante. Desde nuestro enfoque, es una perspectiva curricular que encierra complejidad e interés; innovadora, si bien, cargada de incertidumbres, incógnitas y cuestiones abiertas. El propósito del artículo, dividido en tres partes, es doble: presentar una síntesis muy breve e invitar, a la vez, al estudio reflexivo y crítico en torno al enfoque de competencia asumido por la Institución. En esta primera parte, con acento teórico conceptual, me propongo argumentar la enorme problematicidad del término competencia en el contexto educativo, en especial en el superior. La referencia bibliográfica inmediata la constituyen cuatro documentos: Plan Estratégico de Desarrollo 2012-2019 (Institución Universitaria Antonio José Camacho, 2012), Modelo Pedagógico (Institución Universitaria Antonio José Camacho, 2013), Proyecto Educativo Institucional (Institución Universitaria Antonio José Camacho, 2015) y Enfoque de Competencia (2016). Un concepto propio, innovador, potente y sistémico para los procesos educativos.

\section{DESARROLLO}

Un estudio y debate educativo necesario y provocador, para empezar, tiene que ver con las grandes inquietudes, dudas y cuestiones que ha suscitado la palabra competencia tanto en su inserción en el 
campo laboral como educativo. Una de ellas es la que se refiere a la problematicidad educativa que porta o encubre, la que de alguna manera se ha puesto de manifiesto en la práctica a través del cambiante lenguaje de la educación, en particular de la superior, y la consecuente polisemia del término, llegando hasta el extremo de contar con múltiples acepciones y preferencias personales, junto con la indistinción o confusión conceptual derivadas de todo ello. En definitiva, consideraciones conceptuales críticas como preludio, justificación y acceso a un nuevo abordaje.

Se inicia compartiendo las reflexiones críticas del autor sobre el asunto (una de mis preocupaciones educativas) realizadas desde 1995, una vez se implantaron en el mundo, incluido Colombia, determinadas por organismos internacionales como la OIT, CINTERFOR, Proceso y Declaración de Bolonia (1999), el Proyecto Tuning-Europa, introducido en el proceso La Sorbona-Bolonia-Praga-Berlín, mediante el cual se instauró un espacio de educación superior integrada en Europa, en el trasfondo del área económica europea, léase modelo de desarrollo neoliberal, y el fracasado Proyecto Tuning-América Latina (2005). Un hecho de importancia para destacar aquí es el relacionado con la Organización para la Cooperación y el Desarrollo Económico (OCDE, 1961), un organismo mundial denominado de cooperación internacional, que viene emitiendo, para sus países miembros (y donde Colombia ya forma parte de dicha organización), entre otras, directrices y políticas en materia educativa como es el caso de las pruebas PISA, en el contexto del Programa Internacional para la Evaluación de Estudiantes, lo que explica en parte y muy especialmente, el impulso dado al enfoque de la educación por competencias, más allá de las razones que buscan persuadir sobre la relevancia que puede tener el desarrollo de competencias en un mundo globalizado y complejo como el que vivimos; exigencias que sobre todo responden, como se puede observar, a organismos internacionales quienes vienen requiriendo esta clase de modelo de formación. Desde donde se puede colegir que las dos propuestas sobresalientes en el mundo, que izan y jalonan la formación por competencias emergen en Europa, primero con el proyecto Tuning, impulsado por la Unión Europea y luego el proyecto Definición y Selección de Competencias (DeSeCo, 2000 y 2005), que promueve la OCDE, el que plantea las siguientes competencias: matemática, en ciencia y tecnología, comunicación en la lengua materna y comunicación en una lengua extranjera, digital, en aprender a aprender, interpersonales y cívicas, espíritu emprendedor y expresión cultural (Moreno O. T., 2010).

Siguiendo la misma idea, en el marco de la globalización, se asimila competencia con competitividad, sobre todo cuando se examinan los fines del mercado internacional y se halla que ubican a la educación como una mercancía, junto con las consecuencias de toda índole que ello ocasiona. Al revisar la historia de las propuestas de los diferentes organismos internacionales, parece que preexiste un interés marcadamente economicista que educacional, al no encontrarse un equilibrio entre la educación con miras al desarrollo humano, para la vida y la capacitación centrada y reducida a las necesidades inmediatas de la industria para la competitividad, para el empleo, al nivel tanto nacional como trasnacional. En este orden de la discusión, se descuida todo aquello que se refiere a la pertinencia del aprendizaje según los diferentes entornos socioculturales (contextos que atribuyen sentido), todo ello en acuerdo hoy con los desarrollos de las teorías del aprendizaje, reunidas en torno del neuroaprendizaje, con basamento en los resultados investigativos logrados ya en este siglo en el campo de las neurociencias.

El otro referente de interés para el objeto de la reflexión crítica que se realiza es el Manual Operativo CINE, 2011: Directrices para Clasificar Programas Nacionales de Educación y Certificaciones Relacionadas, del Instituto de Estadística de la UNESCO. Este trabajo se orienta hacia la Normalización y Estandarización mundial de la educación en el marco de la Globalización de la economía, para el caso, según el organismo mundial OCDE. 
Y en esta construcción de antecedentes es útil reconocer que la palabra que nombra el concepto competencia, en su desarrollo histórico y alrededor de su uso ideológico y/o acrítico instalado ya en el campo educativo, donde cobra interés inicialmente en el campo disciplinar de la lingüística, con el aporte de Chomsky, N. $(1965,1977)$ de la competencia lingüística como capacidad de un hablante-oyente ideal, referido sólo a procesos de pensamiento y desarrollo lingüístico y que después es trasplantado al dominio de lo laboral en el ámbito de las denominadas competencias laborales. Sin bien es cierto, que en el rastreo de la génesis del concepto se puede retroceder y llevarla hasta Aristóteles (Metafísica, 2018, p. 202-203) en su distinción y oposición que estableció entre potencia y acto, como algunos antecedentes filosóficos del concepto (Bustamante Z., G., 2002, p. 150-153, 2003, p. 29-42).

Ahora bien, en lo que toca con Colombia. Primero, su ingreso se hizo a través del SENA (1995-1996), con las denominadas competencias laborales, y posteriormente, después del 2000, el término competencia se expande en el sistema educativo mundial, incluido obviamente el colombiano, prácticamente prescritas, sin ninguna fundamentación, contextualización educativa y el necesario debate académico, para derivar en la moda de las "competencias", en lo que se viene llamando educación por competencias, y donde las "competencias", tal como han sido fijadas, nada tienen de innovador. En la Institución es objeto de examen, primero durante el diseño y desarrollo del Proyecto Papyrus (2008-2010), y luego desde el 2012 una vez se abordó la elaboración del Modelo Pedagógico (2013: 43-45) por un colectivo de trabajo interdisciplinario y más recientemente, en el 2017, con el proceso de Resignificación Curricular, el que tomó como uno de sus referentes el Enfoque de Competencia asumido por la UNIAJC (2016).

En acuerdo con Gallego R. (2000, p. 7-8), entre otros profesores-investigadores colombianos $y$ extranjeros como Barnett, R. (2001), Bustamante, G. (2002), Díaz, A. (2005), Gimeno, J. (2009, 2010) y De Zubiría, J. (2014): ¿Tiene sentido sostener la idea de que las competencias constituyen un problema educativo? ¿En qué sentido es un problema educativo legítimo? Y ¿Qué tienen de novedoso, si es que lo tienen? La respuesta a la primera cuestión es afirmativa. Lo es, en principio, porque simplificar y reducir lo que con el uso de la palabra se quiere significar poniéndola en una definición escueta (en dos o tres líneas, por ejemplo), ostenta de manifiesto declararse indiferente frente a todo rigor conceptual y debate académico, una vez ubicados en el campo de las ciencias, incluidas las neurociencias, las de la vida, de la complejidad y obviamente las de la educación, junto con su consecuente profundización filosófica, curricular e ideológico-política (Apple, 2008, p. 11-40, 85-138), en lo que se puede denominar una revisión de sus fundamentos mediante la discusión racional y crítica de conceptos (empleo de la estructura de pensamiento conceptual, Brito J. G. 2014, p. 26-33); también del pensamiento formal y categorial (De Zubiría S. M., 1996) y categorías.

En lo pertinente, el problema del concepto es uno de los más relevantes de la teoría del conocimiento. Al respecto, recordemos que: todo concepto tiene una historia y es concepto pero en una red conceptual o, en otras palabras, en una imbricación conceptual; valga reafirmar, no es aislado, no se reduce a una definición, no hay concepto simple, todo concepto tiene componentes, se enlaza con otros vecinos y se determina en tal sistema de interrelaciones; todo concepto remite a un problema (Zambrano, A., 2005, p. 21-30); los conceptos son los átomos o las partes con las que se construyen las proposiciones. Más aún, tratándose del concepto científico, este es el componente lógico central en la construcción de los sistemas explicativos de cada ciencia, que emerge como producto de un laborioso y arduo trabajo de abstracción y generalización activa del razonamiento humano; el concepto es siempre la "unidad de constituyentes opuestos", que ponen de manifiesto los aspectos contradictorios de las mismas cosas, procesos o fenómenos en su unidad/diversidad, interacción y complementariedad, igualmente el papel 
de los conceptos científicos en el conocimiento del mundo y en la actividad práctica de las personas (Kursanov, G. A, 1966, p. 9-10, 209-286); a su vez, se insiste en la función tanto cognoscitiva como práctica del concepto durante el proceso de construcción del conocimiento, consistente en el difícil y lento tránsito que realiza la razón humana del fenómeno hacia la esencia (aspecto a tratar en cierto detalle más adelante), y en su importancia definitiva en el desarrollo de las ciencias sociales y humanas, de las ciencias de la naturaleza y de las matemáticas. Aquí, como mediadores, representadores y organizadores ideográficos, son útiles el mapa conceptual, la red conceptual, la red semántica, el mentefacto conceptual, entre otros.

En este marco referencial, es atrayente considerar lo relacionado con complejidad y complejización -en ciencias de la complejidad- lo que se diferencia notablemente de simplista, inmediatista e ingenuo, de compartimentación, fragmentación o segmentación del fenómeno que se estudia, al reducir-elaborar la explicación en términos atomizados, compositivos, o agregativos (Maldonado, C. E., 2016, p. 23-41). No obstante, es necesario, como punto de "llegada" y no de "partida" en un proceso investigativo, respecto a la comprensión y tratamiento del objeto de estudio, recurrir a una demarcación metodológica, que siempre se hace forzosa pero provisoria en la construcción del camino prolongado, zigzagueante y difícil que sigue el conocimiento mediante el razonamiento humano en la búsqueda de la esencia (el trasfondo) oculta(o) del fenómeno.

Y avanzando un poco más en la comprensión que se intenta desarrollar y sustentar, ya desde la óptica de la pedagogía conceptual, formando parte de las ciencias de la educación, un concepto es un nodo de proposiciones (Brito, J. G., 2014, p. 29), donde el sujeto, $\mathrm{S}$, de las oraciones que designan tales proposiciones (ideas con sentido completo subyacentes a cada una de las oraciones, que como estructuras gramaticales tienen sujeto, $\mathrm{S}$, y predicado, $\mathrm{P}$ ), es común a todas ellas; este sujeto, $\mathrm{S}$, es el núcleo o el "centro" de atracción y conexión de cuatro va- riedades de oraciones, las que permiten la configuración del representador-organizador ideográfico (ideograma) denominado mentefacto conceptual (otro instrumento bastante útil, en este caso, para mediar, representar y organizar gráficamente, de manera esquemática, la estructura interna de un concepto) (De Zubiría S. M., 1996). El mentefacto no simboliza, representa el concepto tal y como aparece en la mente/cerebro de un sujeto concreto y determinado; el nombre del concepto aparece, entonces en la parte central del mentefacto.

Pero ¿para qué el énfasis, quizás incurriendo en la redundancia, al insistir en la importancia o potencia del concepto, del concepto científico, en el ámbito de las ciencias? Para generar, en la segunda parte del artículo, las condiciones dialógicas que posibiliten contextualmente el desarrollo argumental del concepto <<competencia $>$, creado y propio de la UNIAJC; que como un aporte y componente lógico central en el campo de los estudios curriculares en el país (Montoya, J., 2016), y también como una reflexión crítica sobre la práctica educativa (Gimeno, J., 1998), orienta el diseño, el desarrollo, la implementación y la evaluación de los diferentes programas formativos acordes con la concepción y modo de tratar (enfoque) el problema educativo conexo, de tal manera que nos permita abrir la perspectiva y captar las complejidades con las que debemos tratar.

Prosiguiendo, ahora desde otro punto de vista complementario con los anteriores, el del conocimiento como red, al entender una cosa, un proceso o un fenómeno, objeto de estudio, como una red de redes de relaciones colaborativas, nuestras descripciones o explicaciones constituyen también una red interconectada de conceptos y modelos (Capra, F., 2015, p. 56-62), en lo que se viene mencionando como el pensar sistémico (Capra, F., 2015, p. 56-58; Garciandía, J. A., 2005, p. 131-139). No olvidemos que hablar de sistema nos conduce ineludiblemente a la idea de relación y, desde su estructura y dinámica, bajo la topología de red (Topología y teoría de redes; Maldonado, C. E., 2016, p. 116-132), los diferentes ideogramas permi- 
ten poner en evidencia (visualizar) tal sistema de relaciones, puesto que establecen un vaso comunicante, una especie de túnel, un puente, un eslabón mediador adecuado; lo que es mucho más difícil de lograr directamente sin ningún tipo de mediación, en un discurso científico escrito o hablado, el que obedece justamente a una secuencia de palabras, de oraciones y de párrafos, coherente con la estructura lineal de dicho lenguaje, lo que puede contribuir, de una manera importante, al detrimento de la comprensión profunda, esto es, de la esencia, si se tiene en cuenta la relación de unidad fenómeno/esencia, donde: el fenómeno es aquello que emerge, lo que aparece, lo visible, el aspecto externo, el síntoma, lo que se presenta en forma directa, la forma en que la esencia se expresa; y la esencia es lo subyacente, lo interno, lo que permanece oculto tras la superficie del fenómeno, lo que se presenta en forma indirecta, el trasfondo; en fin, lo que produce y explica el fenómeno.

A destacar en esto último es el carácter contradictorio y complementario de la unidad del fenómeno y la esencia. Por ejemplo, así en el caso del fenómeno del rayo (relámpago y trueno) y su explicación desde la física, donde coexisten en unidad y lucha de opuestos lo visible y lo oculto, lo directo y lo indirecto, lo superficial y lo profundo; igual sucede con los fenómenos cuyo objeto de estudio los abordan la sociología, la biología, la química, la psicología, las ciencias de la salud, las ciencias empresariales, las ingenierías, las ciencias de la educación, entre otras; fenómenos, donde sobre todo está inmersa la condición humana, en su mayoría muy complejos, tales como los sociales, de la salud, los educativos, que exigen un abordaje interdisciplinar y más aún, transdisciplinar, el nivel máximo de integración, donde se borran las fronteras disciplinares (Ander-Egg, E., 1994, p. 13-24; Uribe, C. \& Núñez, J., 2012, p. 26-57). Así mismo, es interesante anotar, en este contexto amplio de reflexión crítica, la relación de unidad, lucha de opuestos y complementariedad entre las ciencias sociales-humanas, las ciencias de la naturaleza y las ciencias formales (matemáticas y lógicas formales), una contradicción dialéctica esencial y, por lo tanto, necesaria de enfrentar y tratar, en específico, en el ámbito de las ciencias de la educación, tanto en los procesos investigativos como formativos, junto con la consecuente producción de conocimiento (Lévy-Leblond, J-M, 2002, p. 11-49), y la ineludible inclusión del sujeto y el diálogo de saberes.

Ahora bien, aquello de las redes conceptuales, del conocimiento como red, del pensamiento no lineal, de los sistemas dinámicos no lineales, del pensar sistémico, del pensar complejo, no debiera surgir como algo en absoluto extraño, extravagante, rebuscado o anormal, puesto que encuentran su correspondencia causal-circular virtuosa con las redes neuronales biológicas (¡la conexidad de miles de millones de neuronas!), el soporte material de los procesos mentales: así, las redes conceptuales implican las redes neuronales biológicas y, a su vez, las redes neuronales biológicas implican las redes conceptuales; todo ello en completo acuerdo con la unidad inseparable de la relación compleja cerebro/mente/cuerpo/entorno (como una totalidad indivisible) (Moriello, S., 2013); dado que los procesos mentales, en última instancia, son procesos cerebrales (Puche, R., 2009; Bartra, R., 2010; Kaku, M., 2014; Izaguirre, M. H., 2017; Caicedo, H., 2017; Toro, J. \& Yepes, M., 2018; Llinás, R. 2018); lo que parece formar parte integrada del fenómeno físico del entrelazamiento (el que funciona también en sistemas biológicos) existente en el universo (según la Física y la Cosmología actual), donde todo está conectado, el que consiste en la superposición de estados al nivel cuántico (lo relacionado con el comportamiento de la materia en el mundo y submundo de lo molecular-atómico, a escalas y dimensiones muy pequeñas, de lo micro o lo nano) en un sistema compuesto (complejo) organizado en dos o en muchos más subsistemas (y donde no se habla ya de <<aquí o allá $>>$ sino de <<aquí y allá >> (Aczel, A. D., 2004, p. 11, 40, 218). Conviene destacar aquí los extraordinarios avances en el conocimiento que hoy se tiene en el tránsito que se realizó desde finales del siglo pasado, de la biología celular a la biología molecular: un salto cualitativo excepcional y gigantesco, con implicaciones directas en las neurociencias y, sobre todo, 
en el neuroaprendizaje en particular, orientadas a descifrar su naturaleza como fenómeno humano y educativo muy complejo, aún en investigación y por mucho más tiempo.

En fin, centrándonos ya en el propio objeto de la discusión que se trae, la transformación del contenido/forma de la estructura en red de la mente/cerebro no lineal al contenido/forma de la estructura lineal del lenguaje hablado o escrito y viceversa, es un proceso muy complejo que necesita la mediación adecuada, la transposición didáctica, tal como ya lo hemos anotado.

Esto de la indiferencia a todo rigor conceptual y debate académico en la universidad, en lo atinente al objeto conceptual (constructo, entendido como creación mental/cerebral) designado por la palabra competencia, parece encontrarse inmersa en la actitud inmediatista y facilista a la que ha conducido la necesidad permanente de su concreción y operacionalización, a lo que cueste, sin más; entonces ¿por qué y para qué hacerlo así? ¿qué se quiere ocultar o soslayar? Indiscutiblemente, la concreción y operacionalización son necesarias en educación, pero no a cualquier costo, allí donde todo vale. Lo es también, porque no deben constituirse en palabras con las que se quiere expresar, de otro modo, aquello que se designa o formula con objetivos o logros; o, de otra parte, cuando todo el trabajo educativo se ha limitado simplemente a cambiar en el discurso la palabra competencia por inteligencia; la palabra competencia por capacidad, por aptitud o habilidad, por destreza o por conocimiento práctico,...; por ejemplo, al sustituir la capacidad para trabajar en equipo por la competencia para trabajar en equipo, el compromiso ético por la competencia ética, las habilidades interpersonales por las competencias interpersonales; ¿en dónde está, entonces la novedad, el aporte significativo a la evaluación de la calidad de la educación? ¿Qué tienen de específico? ¿En qué consiste su solvencia empírico/conceptual o práctico/ teórica? ¿Qué implicaciones han tenido, respecto al cambio, en las diferentes prácticas educativas? (Gimeno, S., 2009, p. 15-58; 2010, p. 328-332). ¡No es la primera vez que una palabra en educación irrumpe allí y se pone de moda!, presentándose la reducción a un comodín verbal o rótulo con su consiguiente desgaste.

En general, el movimiento, la evolución y la fuente o motor del desarrollo en lo tocante con las instituciones educativas se explica en parte por la presencia de al menos tres contradicciones dialécticas (o tensiones, palabra muy utilizada en el lenguaje educativo cotidiano) esenciales: cultura institucional/cultura académica, lo concreto/lo abstracto y adaptación/pensamiento crítico/cambio, donde el sobrepeso desequilibrante se coloca, con bastante frecuencia, del lado del componente cultura institucional, del componente lo concreto y del componente adaptación; lo que se aprecia, referido de una manera prosaica así:

(...) ¡dígame en concreto, de una manera precisa, en tres o cinco palabras, lo que significa el término!, (...) -nótese aquí un requerimiento cercano a lo trivial, a lo simple-, (...) ¡explíqueme qué es lo que hay que hacer al respecto!, (...) ¡es una manera de enredar o complicar lo que está desenredado!, (...) o, (...) ¡esos términos tan rebuscados! (...) desconociéndose, de una manera extraña, que todo campo disciplinar, cualquiera que sea, tiene su lenguaje (por lo tanto, una gramática) y utiliza sus propios términos técnicos; donde dichos términos nombran o designan conceptos o procedimientos fundamentales en el respectivo campo disciplinar y no en otro. Significativo: cada disciplina científica se caracteriza, entre otras cosas, por una serie interconectada de principios, conceptos, categorías y leyes que constituyen un sistema definido, y como sistema lógico es el resultado del desarrollo histórico del conocimiento de la parcela o esfera concreta de la realidad que se estudia. (Kursanov, G. A., 1966, p. 280)

Dicho desequilibrio constituye un obstáculo epistemológico, siguiendo a Bachelard, G., (1978, p. 15-26), con compromisos que bloquean la transformación educativa de la organización y traban, por lo tanto, los procesos investigativos, al colocar mecanismos -inconscientes o no- de defensa 
al cambio, siempre presentes en una dinámica de sucesivos replanteamientos teórico/prácticos implicados; como por ejemplo, en el proceso de resignificación curricular propio de la UNIAJC, conducente a la atribución de nuevos sentidos a las prácticas educativas en todos los niveles e instancias académicas, administrativas, de planeación y operación en la Institución. Resignificación, entendida como un proceso complejo de ruptura crítica con unas prácticas a las que subyace, de todas maneras, un saber previo-tradicional que las soporta y explica; cambio que emerge al tratar la contradicción dialéctica deconstrucción/reconstrucción de significados nuevos.

Ahora bien, para que las competencias constituyan un problema educativo genuino, objeto de estudio, de investigación, necesariamente han de formar parte estructural de una configuración o cuerpo conceptual y metodológico (Gallego, R., 2000, p. 7-8), tal es el caso de una teoría y práctica (teoría/ práctica) curricular.

Y en lo que toca con la novedad que podrían aportar las competencias, es útil resaltar aquí lo siguiente: el enfoque o manera de concebir y tratar la cuestión educativa coligada se ubica justamente en la posibilidad de cambiar de perspectiva desde la cual se lean, se reflexionen críticamente y se comprendan de otro modo los procesos curriculares comprometidos; una perspectiva diferente a la tradicional; visión arraigada y aún dominante, esta última, que entre otros inconvenientes, compartimenta lo real y como consecuencia fragmenta el conocimiento en campos disciplinares estancos, hiperespecializados (materias o asignaturas tratadas de manera aislada y vigilantes de sus respectivas fronteras), hegemónica, homogenizante, universalizante y eurocéntrica, que son algunos rasgos de las prácticas curriculares actuales que predominan en el mundo globalizado neoliberal (II. Globalización y educación. Mejía, M., 2006, p. 83-174; Morin, E., 2010, p. 9-16, 83-98); al contrario, se trata de un rumbo alterno, un paradigma emergente, innovador, sistémico, por lo tanto, integrador e incluyente, conducente a decolonizar, a rea- lizar aportaciones idóneas y endógenas orientadas desde el Sur hacia la reinvención de la educación (Morin, E., \& Delgado, C. J., 2018), una necesidad, un imperativo en este siglo XXI. Y, en este nuevo contexto educativo, necesitamos la formación de profesionales reflexivos y críticos dispuestos a enfrentar y tratar las cegueras y brechas del conocimiento, la condición humana (¡muy compleja!), las incertidumbres, las contradicciones dialécticas, la borrosidad conceptual (Kosko, B., 1995, p. 15-72, 154-224), lo probable/lo improbable, los obstáculos,...; en fin, las múltiples y enormes dificultades que nos presenta este siglo XXI: un mundo, una región, un país mutante, convulsionado(a), en permanente crisis (Morin, 2001, 2010, 2018).

Se trata entonces ahora de concretar en la praxis curricular, de poner en acción lo concebido, lo propuesto; en suma, de poner manos en la obra que nos ocupa, donde una especial relación de unidad/diversidad y complementariedad a tratar con dificultad y tesón es: macrocurrículo/mesocurrículo/currículo/microcurrículo/plan de curso/lo vivido en el aula; resaltando la complejidad de los procesos formativos en el aula de clase.

¡Una especial invitación a estudiar, a investigar, a conocer el conocer, a caminar en colectivo institucional abriendo camino! Hemos de enfrentar y resolver múltiples problemas de complejidad creciente que se plantearán a todo lo largo de los procesos de diseño, desarrollo e implementación curricular de los diferentes programas de formación profesional, en todos los niveles e instancias de la organización educativa en la UNIAJC. Uno de los más críticos es el relacionado con la evaluación.

Para cerrar esta Primera parte, no dejemos pasar por alto que la identidad del ser humano -muy compleja por cierto- es tanto biológica, como cultural, histórica, social y psicológica; identidad entendida y tratada como lo uno, y a su vez, múltiple, esto es como: unidad/diversidad y complementariedad, lo que interconecta las relaciones individuo/sociedad/especie, cuerpo/cerebro/mente/ cultura, razón/afectividad/pulsión/locura, entre 
otras (Morin, E., 2009, p. 27-74; Capra, F., 2015, p. 56-63; Maturana, H., 2002, p. 91-107, 245-259), atribuyendo el pleno sentido de lo humano, esto es, el ser del ser humano y que, por lo tanto, no conlleva su fragmentación, lo que tiene que ver, formando parte constitutiva, en general, de toda la organización de lo vivo (Maturana, H. \& Varela, F., 1996: XXV, 5-35); que todo el conocimiento es, a la vez local y total (De Sousa S., 2009, p. 47-50), ello en acuerdo con las relaciones complejas entre el todo y las partes y, a su vez, entre estas, ubicados ya en el contexto científico y educativo del tercer milenio. Y que finalmente el Enfoque de Competencia propuesto, constituyendo parte de la formación y el desarrollo a escala humana (Max-Neef, M. y otros, 1996), para la vida, para la paz, no es la panacea, no es la receta o respuesta solucionadora sacada de la manga, por el contrario, es como un faro que ilumina y constituye una apuesta educativa innovadora (la que necesita, a su vez, del recurso a la estrategia, Morin, 2001, p. 94-96), una hipótesis de trabajo curricular plausible, que como tal no ostenta el nivel de solución definitiva, la que implica, entre otras cosas, un sendero que no existe pero que se construye colectivamente al caminar, en el juego de las posibilidades y contradicciones, navegando en un océano de incertidumbres a través de archipiélagos de certeza (Morin, E., 2001, p. 76-78, 90-92); puesto que es en la incertidumbre y unidad/diversidad/diálogo/complementariedad de (entre) las contradicciones esenciales donde habitan la autonomía/dependencia y, por lo tanto, la ocasión para innovar y crear. A la universidad, a la UNIAJC, le tocará jugar un importante rol en estos momentos de crisis (Zabalza, M. A., 2011).

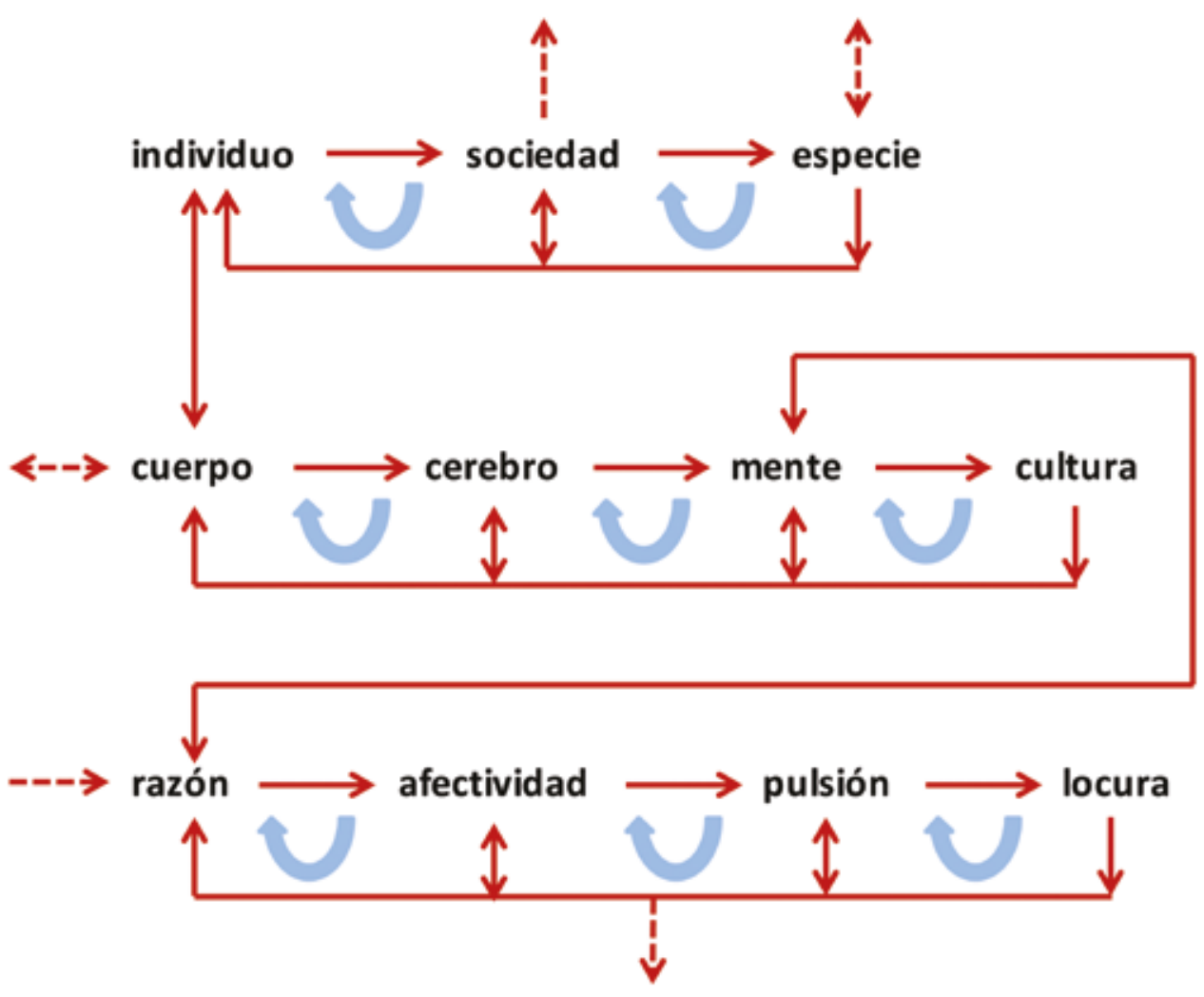

Fig. 1. Redes de redes de relaciones colaborativas Fuente: Elaboración propia (2019) ${ }^{1}$.

Una síntesis bajo representación gráfica, en complementariedad, con fundamento en los aportes de los siguientes referentes bibliográficos e investigativos: Morin, E., 1988, 2009; Capra, F., 2003, 2015; Maturana, H., 2002; Aczel, A. D., (2004); Torp, L. \& Sage, S., 2007; Moriello, S. A., 2013; Maldonado, C. E., 2016; Uribe C., H., en Hernández R., C. A., y otros, 2017 
La figura 1 visualiza las redes de redes de relaciones colaborativas (bucles o ciclos recursivos) que caracterizan el subsistema funcional en consideración, el que integra otros y, a su vez, forma parte de otros más amplios que lo contienen e interactúan con él y con otros; donde, por lo tanto, no hay partes aisladas; es "el cambio de esquemas de objetos a relaciones” (Capra, F., 2015, p. 56-62). Obsérvese como la red está constituida por redes y a su vez forma parte de redes cada vez más abarcativas e integradoras.

En la segunda parte del artículo se abordará en detalle el Enfoque de Competencia -del que forma parte integrada el concepto $<<$ competencia $>$ asumido por la Institución, junto con las profundas implicaciones que tiene en las prácticas educativas en todos los niveles e instancias académicas, administrativas, de planeación y operación, tanto en el nivel meso como micro curricular, en especial, en el proceso de enseñanza y aprendizaje y, sobre todo, en la evaluación, la que deberá cambiar drásticamente de perspectiva. De todas maneras, se trata, por ahora, de unos avances, de unas elaboraciones parciales, en lo que respecta a la producción del conocimiento necesario en el trasegar sinuoso por el "mapa inacabado de la complejidad" (De Almeida, M. D. C., 2008, p. 9-12). La razón que me ha llevado a escribir este artículo, dividido en tres partes, es educativa, por la luz que nos puede arrojar al compartir inicialmente mis reflexiones críticas sobre la cuestión y, a continuación, sobre el modo de concebir y tratar la competencia propuesta institucionalmente para los procesos educativos en la UNIAJC, con fundamento en la concepción humanista, el pensamiento complejo y la gestión del conocimiento. También he tenido como motivo especial invitar al estudio e investigación educativa que el diseño, desarrollo e implementación curricular conlleva, y a formar parte de comunidades de práctica y redes de conocimiento al nivel tanto nacional como internacional. Finalmente, consideremos la siguiente cita, brillante y apropiada a lo que nos habita: "La partitura es una apuesta en la posibilidad de concretización sonora" (Lima, R. F. En: De Almeida, M. D. C., 2008, p. 11).

\section{CONCLUSIONES}

Hemos encontrado que los desafíos educativos de reconceptualización y metodológicos que nos presenta este siglo XXI en el contexto de la denominada sociedad de la información y el conocimiento son gigantescos; estos requieren prácticamente reinventar la educación, un nuevo modelo de universidad, una vez hemos dimensionado y tomado conciencia de la pertinencia y complejidad del problema que se aborda, reavivándose el debate sobre la misión de la universidad. En crisis currículos enciclopédicos, métodos memorísticos y mecanicistas centrados en procesos educativos, que definitivamente deben trascender sustancialmente de la concepción bancaria del cerebro/mente como depósito de datos e información a llenar, hacia el desarrollo de actitudes, valores y habilidades del pensamiento, incluido el reflexivo crítico, enlazadas con habilidades específicas según determinado campo disciplinar - para la transformación de información en conocimiento-, de la comunicación y el lenguaje, de la segunda lengua, para la innovación y la creatividad, relacionadas(os) con la ética profesional, la estética, el trabajo en equipo, el liderazgo y el emprendimiento, entre otros requerimientos inherentes a los componentes básico, específico y transversal, que se constituyen en parte integrada y conexa del desarrollo de la respectiva competencia profesional. Se trata, entonces, de una educación liberadora, para la vida, para el trabajo, para el ejercicio de la autonomía, la atribución de significados y la colaboratividad.

No existe ni podrá existir acuerdo en el mundo respecto a qué son las competencias y cómo abordar tanto el diseño como el desarrollo e implementación curricular; ello representa una gran oportunidad para asumir posiciones con propuestas innovadoras. Además, el éxito y utilidad de una propuesta educativa no está asegurada por el hecho de provenir de organismos internacionales, gubernamentales o intergubernamentales; tampoco lo es desde el enfoque que se adopte, sin importar, inclusive, que esté bien fundamentado; naturalmente, y de todas maneras, instaura sí una 
apuesta educativa innovadora, una hipótesis de trabajo curricular plausible, donde innovar implica asumir ciertos niveles de riesgo.

Las competencias constituyen un problema educativo legítimo y la formación con enfoque de competencias puede ser innovadora si se cambia de enfoque o de paradigma en comparación con el abordaje tradicional, que se ha caracterizado por ser prácticamente un disfraz de cambio y no una alternativa a las propuestas existentes hoy en día. En este sentido, configuran un campo de estudio, de investigación educativa; por lo tanto, el desarrollo e implementación curricular con enfoque de competencia se debe tratar como tal, mediante la reflexión crítica en la acción.

\section{BIBLIOGRAFÍA}

Aczel, A. D. (2004). Entrelazamiento. Madrid: Drakontos Crítica

Ander-Egg, E., (1994). Interdisciplinariedad en educación. Buenos Aires: Editorial Magisterio del Río de la Plata

Apple, M. W. (2008). Ideología y currículo. Madrid: Ediciones Akal, S. A.

Aristóteles (2018). Metafísica. Barcelona: Plutón ediciones X, s, I. Tercera Edición

Bachelard, G. (1978). La formación del espíritu científico. Buenos Aires: Siglo XXI Editores S. A.

Barnett, R. (2001). Los límites de la competencia. El conocimiento, la educación superior y la sociedad. Barcelona: Editorial Gedisa, S. A.

Bartra, R. (2010). Antropología del cerebro. La conciencia y los sistemas simbólicos. México D. C.: Fondo de Cultura Económica. Pre-Textos.
El enfoque de competencia de la UNIAJC constituye una hipótesis de trabajo curricular alternativa, innovadora y plausible que orientará las transformaciones educativas requeridas en la Institución; pero demanda trabajo en equipo interdisciplinar.

Hay un enorme interés y preocupación por el conjunto de condiciones concretas, de toda índole, que se necesitan para realizar las transformaciones inquiridas dado el nivel de complejidad del problema educativo que se acomete, el que se manifiesta en todas las instancias institucionales y gubernamentales, y que hacen posible o no su viabilidad transformadora en la organización; por lo tanto, en la praxis educativa y creadora, entendida esta no sólo como reflexión e interpretación del acto educativo, sino como criterio de validez y derrotero de su propia transformación.

Brito A., J. G. (2014). Pensamiento conceptual y pensamiento crítico. Revista Internacional Magisterio (66).

Bustamante Z., G., y otros. (2002). El concepto de Competencia II. Una mirada Interdisciplinar. Bogotá, D. C.: Sociedad Colombiana de Pedagogía. Alejandría Libros.

Bustamante Z., G. (2003). El concepto de Competencia III. Un caso de recontextualización. Las "competencias" en la educación colombiana. Bogotá, D. C.: Sociedad Colombiana de Pedagogía. Alejandría Libros

Caicedo L., H. (2017). Neuroaprendizaje. Una propuesta educativa. Bogotá, D. C.: Ediciones de la U.

Capra, F. (2015). La trama de la vida. Una nueva perspectiva de los sistemas vivos. Barcelona: Editorial Anagrama, S. A.

Capra, F. (2003). Las conexiones ocultas. Implicaciones sociales, medioambientales, económicas y biológi- 
cas de una nueva visión del mundo. Barcelona: Editorial Anagrama, S. A.

Chomsky, N. (1977). Problemas actuales en teoría lingüística. Temas teóricos de la gramática generativa. Madrid: Siglo XXI Editores.

De Almeida, M. D. C. (2008). Para comprender la complejidad. México, Hermosillo, Sonara: Multiversidad Mundo Real Edgar Morin, A.C.

De Sousa S., B. (2013). Una epistemología del SUR: la reinvención del conocimiento y la emancipación social. México, D. F.: CLACSO Coediciones, Siglo xxi Editores, S. A. de C. V.

Delors, J. (1996). La educación encierra un tesoro. Informe a la UNESCO de la Comisión Internacional sobre la educación para el siglo XXI. Madrid: Santillana Ediciones, UNESCO.

De Zubiría S., M. (1996). Instrumentos y Operaciones Intelectuales: Aplicaciones al aula. Mentefactos II. Módulo 5. Santafé de Bogotá: Fundación Alberto Merani para el desarrollo de la inteligencia.

De Zubiría S., J. (2014). Cómo diseñar un currículo por competencias. Bogotá, D. C.: Cooperativa Editorial Magisterio

Díaz B., Á. (2005). Enfoque de competencias en la educación. ¿Una alternativa o un disfraz de cambio? México, D. F.: UNAM. Claves. 7. Perfiles Educativos. Documento PDF obtenido de: https://www.redalyc.org/articulo.oa?id=13211102

Díaz B., Á. (2003). Currículum. Tensiones conceptuales y prácticas. Revista Electrónica de Investigación Educativa, 5 (2). Obtenido de: http://redie.uabc.mx/ vol5no2/contenido-diazbarriga.html

Freire, P. (2005). Pedagogía del oprimido. México, D. F.: Siglo XXI. Editores, S. A.
Freire, P. (2009). Pedagogía de la autonomía. Saberes necesarios para la práctica educativa. México, D. C.: Siglo XXI Editores, S. A.

Gallego B., R. (2000). El problema de las competencias cognoscitivas. Una discusión necesaria. Bogotá, D. C.: Universidad Pedagógica Nacional.

Gallego B., R. \& Pérez M., R. (2003). El problema del cambio en las concepciones epistemológicas, pedagógicas y didácticas. Bogotá, D. C.: Universidad Pedagógica Nacional.

Garciandía I., J. A. (2005). Pensar sistémico. Una introducción. Bogotá, D. C.: Editorial Pontificia Universidad Javeriana.

Gimeno S., J. (1998). El currículo: una reflexión sobre la práctica. Madrid: Ediciones Morata, S. L.

Gimeno S., J. (2009). Educar por competencias, ¿qué hay de nuevo? Madrid: Ediciones Morata.

Gimeno S., J. (2010). Saberes e incertidumbres sobre el currículum. Madrid: Ediciones Morata.

González, J. P., Reyes, R., y otros. (2003). La transformación educativa. Consideraciones. La Habana, Cuba: Editorial Pueblo y Educación

Hernández R., C. A. y otros. (2017). Interdisciplinariedad: Un desafío para transformar la universidad del siglo XXI. Santiago de Cali: Programa Editorial. Universidad Autónoma de Occidente

Institución Universitaria Antonio José Camacho. (2012). Plan Estratégico de Desarrollo 2012 -2019. Cali: UNIAJC.

Institución Universitaria Antonio José Camacho. (2013). Modelo Pedagógico Institucional. Cali: UNIAJC.

Institución Universitaria Antonio José Camacho. (2015). Proyecto Educativo Institucional. Cali: UNIAJC. 
Institución Universitaria Antonio José Camacho. (2016). Enfoque de Competencia. Un concepto propio, innovador, potente y sistémico para los procesos educativos. Cali: UNIAJC

Institución Universitaria Antonio José Camacho. (2017). Proceso de Resignificación Curricular. Cali: UNIAJC

Instituto de Estadística de la UNESCO. (2011). Manual operativo CINE 2011.Directrices para clasificar programas nacionales de educación y certificaciones relacionadas. UNESCO-2015 para la edición española. Recuperado de http://uis.unesco.org/ sites/default/files/documents/isced-2011-operational-manual-guidelines-for-classifying-national-education-programmes-and-related-qualifications-2015-sp.pdf

Izaguirre S., M. H. (2017). Neuroproceso de la enseñanza y del aprendizaje. Metodología de la aplicación de la neurociencia en la educación. Bogotá D. C.: Alfaomega Colombiana S. A.

Kaku, M. (2014). El futuro de nuestra mente. El reto científico para entender, mejorar y fortalecer nuestra mente. Bogotá D. C.: Debate

Kosko, B. (1995). Pensamiento Borroso. La nueva ciencia de la lógica borrosa. Barcelona: Crítica. Grijalbo Mondadori S. A.

Kursanov, G. A. (1966). El materialismo dialéctico y el concepto. México 16, D. F.: Editorial Grijalbo, S. A.

Lévy-Leblond, J-M. (2002). Conceptos contrarios o el oficio de científico. Barcelona: Tusquets Editores, S. A.

Maldonado, C. E. \& Gómez, N. A. (2011). El mundo de las ciencias de la complejidad. Una investigación sobre qué son, su desarrollo y sus posibilidades. Bogotá, D. C.: Editorial Universidad del Rosario.
Maldonado, C. E. (2016). Complejidad de las ciencias sociales. Y de otras ciencias y disciplinas. Bogotá, D. C.: Ediciones desde abajo.

Maturana R., H. (2002). El sentido de lo humano. Santiago de Chile: Dolmen Ediciones, S. A.

Maturana, R., H. \& Varela G., F. (1996). El Árbol del conocimiento. Las bases biológicas del entendimiento humano. Santiago de Chile: Editorial Universitaria.

Max-Neef, M., Elizalde, A., Hopenhayn, M. (1986). Desarrollo a Escala Humana. Una opción para el futuro. Santiago de Chile: CEPAUR.

Mejía J., M. R. (2006). Educación(es) en la(s) globalización(es). Entre el pensamiento único y la nueva crítica. Bogotá, D. C.: Ediciones desde abajo

Montoya V., J. (2016). El campo de los estudios curriculares en Colombia. Bogotá D. C.: Universidad de Los Andes.

Moreno, O., T. (2010). Competencias en educación. Una mirada crítica. México: Revista Mexicana de Investigación Educativa, RMIE. Vol. 15. NUM. 44. Reseña.

Moriello, S. A. (2013). Ciencias de la Complejidad. Una breve introducción. Buenos Aires: Nueva Librería

Morin, E. (1988). El Método III. El conocimiento del conocimiento. Madrid: Ediciones Cátedra, S. A.

Morin, E. (2001). Los Siete Saberes Necesarios para la Educación del Futuro. Bogotá D. C.: Mesa Redonda Magisterio. UNESCO

Morin, E. (2009). El Método. La humanidad de la humanidad. La identidad humana. Madrid: Ediciones Cátedra

Morin, E. (2010). ¿Hacia el abismo? Globalización en el siglo XXI. Madrid: Ediciones Paidós Ibérica. 
Morin, E. \& Delgado D., C. J. (2018). Reinventar la Educación. Abrir caminos a la metamorfosis de la humanidad. Bogotá D. C.: Multiversidad Mundo Real: Ediciones desde abajo.

Puche N., R. (2009). ¿Es la mente no lineal? Cali: Programa Editorial Univalle.

Roldán CH., J., Ben-Dov., Y., y Guerrero P., G. (2004). La Complementariedad: una Filosofía para el siglo XXI. Cali: Programa Editorial Universidad del Valle.

RTVC; Señal Colombia (productores). (2018). Llinás: El cerebro y el universo [Documental]. Colombia: Alianza Colciencias y Señal Colombia.
Toro G., J. \& Yepes S., M. (2018). El cerebro del siglo XXI. Bogotá, D. C.: Editorial El Manual Moderno.

Torp, L. \& Sage, S. (2007). El Aprendizaje Basado en Problemas. Buenos Aires: Amorrortu editores.

Uribe M. C. (2012). La interdisciplinariedad en la universidad contemporánea: Reflexiones y estudios de caso. Bogotá, D. C.: Editorial Pontificia Universidad Javeriana.

Zabalza Beraza, M. (2011). Innovar en tiempos de crisis. REDU. Revista de Docencia Universitaria, 8(2), 9. doi: https://doi.org/10.4995/redu.2010.6192

Zambrano L., A. (2005). Didáctica, pedagogía y saber. Bogotá D. C.: Cooperativa Editorial Magisterio.

\section{AUTOR:}

Hernán Mera Borrero: Asesor pedagógico de la UNIAJC. Se ha desempeñado también como profesor, asesor técnico pedagógico y formador de docentes del SENA; como profesor de Física de la antigua Escuela de Tecnología en Electrónica, y de Física y Geometría en el Departamento de Ciencias Básicas de la UNIAJC. Durante cinco años ejerció como profesor y asesor etnoeducativo en en comunidades indígenas en el norte del Cauca, y cuenta con una experiencia docente acumulada de 50 años. Es coautor de varios libros del proyecto Papyrus y ha participado como ponente en congresos y seminarios nacionales e internacionales. Correo electrónico: hermebo@gmail.com 